\title{
Runtime Semantic Interoperability for Gathering Ontology-based Network Context
}

\author{
John Keeney, David Lewis, Declan O'Sullivan, Antoine Roelens, Vincent Wade, Aidan Boran, Ray Richardson \\ Knowledge \& Data Engineering Group, \\ Department of Computer Science, \\ Trinity College Dublin, \\ Dublin, Ireland. \\ \{John.Keeney I Dave.Lewis I Declan.OSullivan I roelensa I Vincent.Wade\}@ cs.tcd.ie \\ Bell Labs Ireland \\ \{aboran I rayrichardson\}@lucent.com
}

\begin{abstract}
The trends for pushing more operational intelligence towards network elements to achieve more context-aware and self-managing behavior often requires elements to gather network knowledge without necessarily binding explicitly to all of the potential sources of that knowledge. Though event-based publish-subscribe models allow efficient distribution of knowledge where the event types are known globally, dynamic service chains, ad hoc networks and pervasive computing application all introduce a more fluid and heterogeneous range of context knowledge. This requires some runtime translation of knowledge between sources and sinks of network context. This paper builds on existing mapping techniques that use ontological forms of existing management information models to examine the extent to which these can be employed for runtime semantic interoperability for network knowledge. It presents results in developing a management knowledge delivery framework based on existing models and platforms, but which offers a more decentralized knowledge exchange mechanism.
\end{abstract}

Keywords; ontologies, semantic interoperability, content-based networks.

\section{INTRODUCTION}

The management of current telecommunications networks is characterized by a heavy reliance on human operator expertise. Equipment centric network management systems require operators to have a detailed knowledge of how to effect configuration changes, recover from faults, optimize performance, etc. However the increasing complexity of network element interfaces, the highly distributed nature of data, and the growing inter-dependence between network elements is leading to faults being introduced through human error, and more importantly a general decrease in the responsiveness to network events. The backdrop to these developments includes:

- regulatory requirements which require networks to exhibit $99.999 \%$ availability levels - this translates to no more than five minutes down time in a year.
- the emergence of beyond voice media rich services such as VoIP, IM, P-2-T, and IPTV. The time criticality of these new services will lead to the requirement for near real-time response times for fault, configuration, and performance management activities.

There is clear evidence to support the need for a paradigm shift in the approach to managing current and emerging networks. The focus needs to shift from the "How" to the "What" - operators should not need to be aware of the specifics of How to recover from a fault condition or How to implement a configuration change rather they should focus on defining What constitutes acceptable network behavior. Failure to act on this will severely impact the take-up of next generation networks where the How is set to get very much more complex and time critical. Autonomic Communication aims to enable this paradigm shift by migrating management intelligence towards the network elements empowering operational support staff to specify required network behavior in terms of goals and constraints. The envisioned autonomic network will employ a decentralized architecture of peers that intelligently collaborate using dynamically acquired network knowledge to meet business level goals and constraints as set by human network administrators.

A key platform technology for the practical transition to Autonomic Communication is a service that enables the efficient delivery of network operation knowledge to, and only to, nodes that have expressed an interest in that knowledge for use as the context information needed to make appropriate adaptation to network behavior. This paper presents a Knowledge Delivery Service to mediate operational network knowledge in an open ontological form, thereby promoting the graceful evolution of network management applications from contemporary to fully autonomic. To cope with the inevitable heterogeneity of knowledge across the population of network nodes, the service will provide a level of semantic interoperability that will be transparent to the nodes providing and consuming knowledge. The Service will be based on 
content based networking $(\mathrm{CBN})$ principles and will be supported by semantic engineering tools, to provide a practical and comprehensive Autonomic Knowledge Delivery Framework (AKDF).

The framework will provide a vital technology platform for the migration of network management systems from a centralized hierarchical architecture to a distributed mesh of inter-working intelligent agents.

In this paper we present an initial implementation of this framework that combines tools for capturing semantic mappings between different management information models with a run-time CBN environment that integrated with existing network element agents. We provide an assessment of the prospect for useful semantic interoperability mappings being extracted from the current body of management information models. We also present results assessing the performance of the $\mathrm{CBN}$ and an associated management agent gateway against industrial-strength requirements for event monitoring.

\section{BACKGROUND}

The proposed Autonomic Knowledge Delivery Framework represents a novel problem domain at the intersection of: Autonomic Communications and inter-domain network management; knowledge-based network operation support; business driven access control and trust management, and content-based networking. Here we explore these influences in more detail.

\section{A. Autonomic Communications}

Autonomic systems aim to reduce the cost of handling modern ICT complexity by making systems self-managing, i.e. selfconfiguring, self-healing, self-optimizing, and self-protecting. This requires monitoring and analyzing the operational knowledge in ICT systems so that it can be used to plan and execute corrective measures, typically using some artificial intelligence techniques. This relieves the human manager from performing these tasks while allowing the human to guide the decisions made by the autonomic manager through the definition of high-level policy rules defining goals for and constraints on the desired system behavior. The concept has been initially applied to large scale computing systems, where IBM has developed the concept of Autonomic Computing [kephart].

More recently, momentum has been growing to apply autonomic principles to network operations,. An early articulation of the use of operational network knowledge by intelligent applications was proposed by David Clarke et al in a proposal for a Knowledge Plane for the Internet [clark]. Operational network knowledge is defined as network operations or management data accompanied by its meta-data, typically a management information model. A broader autonomic approach has been proposed more recently under the title of Autonomic Communications [smirnov]. Autonomic Communications emphasizes the use of highly distributed forms of automated intelligence such as multi-agent systems, swarm intelligence, or cellular automata [mullany]. All these approaches present a major challenge in obtaining the relevant operational network knowledge that forms the contextual information needed to make adaptive changes to network element behavior. Difficulties arise because the network elements that possess this contextual knowledge are widely distributed, they are purchased from different vendors, they perform different functions, they possess a wide range of knowledge meta-data, and they are operated by different organizations.

\section{B. Knowledge for End-to-end Network Operations}

Deregulation of the telecommunications industry and the commoditization of trunk bandwidth mean that any end-to-end broadband data flow is likely to traverse several IP and optical transport network domains operated by separate and often competing network operators. The challenges in arriving at a practical and durable solution are in addressing crossownership sensitivities, the range of distributed intelligence technologies being investigated, and the lack of related interoperability standards. These present an even more extreme case of the conditions that led to interoperability silos in conventional management systems and thus the looming prospect of Autonomic Communication silos must be quickly addressed. Proposals for end-to-end delivery of operation network knowledge are constrained either to individual protocol layers [thaler] or to following existing signaling paths [schulzrinne]. We therefore propose a novel open and durable solution that combines:

- Semantic interoperability of operational network knowledge using the existing Web Ontology Language (OWL) standards [owl]

- Loose binding between producers and consumers of operational network knowledge

We believe durability is a key requirement for any practical approach for end to end network operations. In order to support the migration from today's, human driven but increasingly complex management systems to one constituted largely by collaborating intelligent agents, any solution must efficiently support either, while also handling the inevitable co-existence of both.

The flow of operational network knowledge end-to-end also presents a problem of access control to knowledge. Operator concerns about the sensitivity and security of operational data is reflected in the hierarchical nature of the manager-agent paradigm and the intra-domain focus of architectures such as TMN [tmn]. The fragmentation of manager-agent protocols at the element layer, and the lack of a dominant interoperation technology at the higher layers has led to problems exchanging management knowledge between vertical silos of interoperability (both syntactic and semantic) within operators' Operational Support Systems (OSS) [adams]. When addressing the exchange of operational knowledge between operators, this is compounded by commercial confidentiality concerns, which result in bilateral agreements and inflexible custom gateways. This spells disaster for the vision of 
Autonomic Communications where intelligence operating at or near the network element level must be able to freely gather contextual knowledge about the state of the network end-toend, while adapting to changes in this context to achieve administrator-specified goals and maintain their constraints. Though we acknowledge this challenge, we do not pursue it further in the paper but have begun to address these issues elsewhere [feeney05].

\section{Semantic Interoperability of Operational Network Knowledge}

Attempts have been made to define new management information modeling and service modeling languages to act as a lingua franca between heterogeneous management models. Notable amongst these are the Distributed Management Task Force's (DMTF) Common Information Model (CIM) schema [cim] and the TeleManagement Forum's NGOSS technology neutral architecture [tmf053]. However, a lack of a strong semantic interoperability mechanism and reliance on conformance to poorly subscribed industrial agreements effectively, demote these to the status of yet further management knowledge formats, with which other schemes needed to interoperate. Recent pioneering work by project Universidad Politécnica de Madrid (UPM) [lopezdevergara04] has shown directly the value of modeling management information models in the OWL ontological format, and how this can be used to ease the interoperation between models originally conceived in different management information languages, i.e., GDMO, SMI, CIM. This also points to the adoption of the OWL-S service semantic language [martin] for defining management services knowledge as well as for supporting to dynamic management service composition. The DIP project (dip.samenticsweb.org) has already investigated the use of the Web Service Modeling Ontology (WSMO) [wsmo] to reduce cost in more rapid development of B2B gateways between different service providers [duke04].

\section{Scalable Delivery of Operational Network Knowledge}

Publish-subscribe systems provide an efficient mechanism for delivering information from its source to one or more interested parties (known as subscribers). When compared to polling approaches, it also allows the timely notification of events or changes to information, but requires publishers and subscribers to agree on the message types before interacting. Content-Based Networks (CBN) extends this approach to allow the subscriber to specify conditional filters on message properties, effectively allowing the subscriber to define the type of message in which they are interested [carzaniga] [segall] [strom]. By delivering operational knowledge only to those knowledge consumers who register a specific interest, while multicasting messages to consumers who share interests offers the potential for scaling knowledge delivery to Internet dimensions [crowcroft]. An alternative approach is to use distributed database techniques, such as been applied in the EDUTELLA platform [loser]. This allows queries to be routed to distributed RDF databases, using a peer-to-peer approach that provides some of the self-configuring features which would be desirable of an autonomic platform. However, it is focused on query-response communication, rather than the publish-subscribe model that better suits the monitoring of ongoing streams resource information, i.e. the primary output of network elements.

\section{AN AUTONOMIC KNOWLEDGE DELIVERY FRAMEWORK}

Agents that collaboratively implement autonomic functions may be arbitrarily distributed across many management application domains. Thus they may not have any knowledge of each other prior to convening to implement an autonomic function. The natural diversity in management knowledge that will exist across the range of network elements and management agents involved in any particular end-to-end communication service must therefore be handled by a Knowledge Delivery Service. The KDS addresses how this network knowledge needed to perform autonomic functions can be gathered efficiently for communications services whose end-to-end delivery spans many heterogeneous network elements. In order to achieve this, the KDS must be able to support semantic interoperability between the model of the knowledge sought by the consumer and that used by its producer. The proposed Knowledge Delivery Service (KDS) will be implemented as a Knowledge Delivery Network (KDN) structured along CBN principles. Thus, network and service elements may advertise the type of knowledge they possess to the KDS while an autonomic manager may place a subscription for the knowledge they need for the task at hand, canceling the subscription when the task is finished. Recent progress in content-based networking has shown that perfect routing can be achieved in a scaleable manner independently of subscriber joins and leaves though subscription aggregation [chand].

Producers of operational network knowledge express their knowledge using an ontological representation of their relevant management information models. These management information models can then be analyzed off-line using semantic matching tools, to produce deployable runtime mappings between instances of these models, which are then exchanged in management notification messages. The advertisement-subscription mapping and subscription aggregation algorithms used in the KDN can then exploit the deployable runtime mappings between different representations of similar management information models to provide a level of semantic interoperability between the autonomic manager and the network elements.

In addition to dealing with the heterogeneous representations of the shared knowledge within the network, the operators of networks in different domains need to carefully control the dissemination of this network knowledge from network monitoring agents and elements to agents in other domains, where these subscribing agents may be completely unknown prior to the initiation of such sharing. Thus access control 
based on shared knowledge of identities, or shared agreement on access control roles, will not be flexible enough to cope with fluid service delivery chains and higher levels of customer-driven management.

The approach taken to the delivery of autonomic network knowledge in this research consists of:

- The use of semantic interoperability based on ontological mappings as a mechanism for handling heterogeneity in management information.

- The extension of content-based networking to use ontology-based advertisements and subscriptions, hence using ontological reasoning for ad hoc matching and filtering inside the network

- The use of ontology-based trust and organizational models to support access control in ad hoc end-to-end business relationships.

In this paper we examine initial results towards the first two areas, while examination of access control will be addressed elsewhere.

This Autonomic Knowledge Delivery Framework (ADKF) consists of the following principal elements:

- A Knowledge Delivery Network (KDN) that delivers knowledge from publishing clients to subscribing clients

- A Knowledge Delivery Service (KDS) through which client applications can access the KDN.

- A set of Tools and Utilities to support the use and operation of the KDS.

An outline of the structure of the AKDF is depicted in Figure 1 below.

\section{A. Modelling Issues}

Ontology-based semantics are used because they support encoding and mapping between separately authored and thus heterogeneous source of knowledge, expressed in a widely accepted standard (W3C's OWL) [owl]. Ontology mapping is defined as the task of relating the vocabulary of two ontologies that share the same domain of discourse in such as way that the mathematical structure of ontological signatures and their intended interpretations are respected [kalfoglou]. In order to generate mappings, various matching techniques can be applied to the ontologies. Several types of matching techniques have been classified [shvaiko]. String, Language, Linguistic, and Constraint -based matching techniques work at the element level of an ontology and are generally classed as "lexical" matchers. Reuse, Graph, and Taxonomy -based matchers are generally classed as "structural" matchers. Finally, Model-based matchers use semantic interpretation (e.g. Description Logics) to find matches and are thus classed

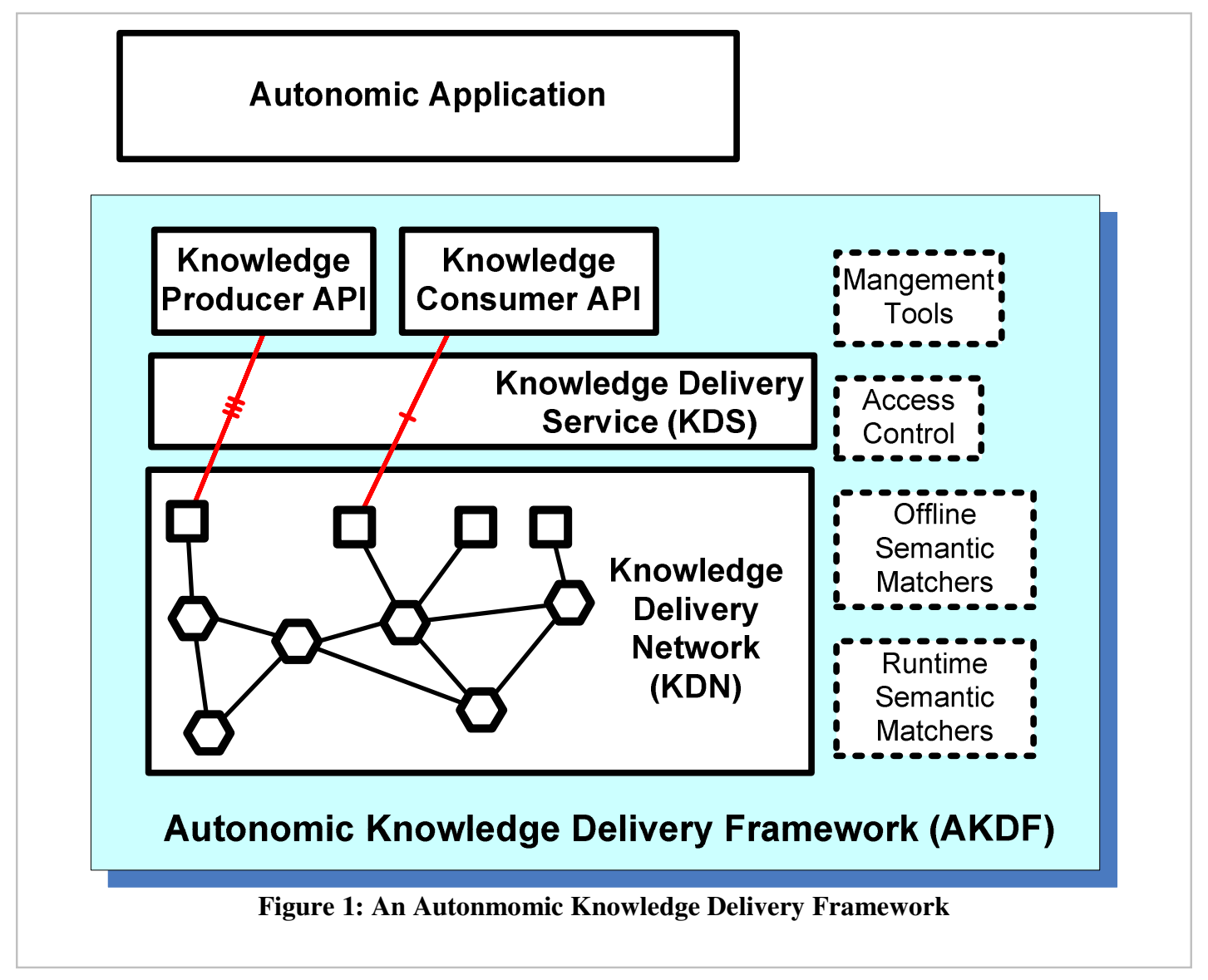


as "semantic" matchers. However, as yet, the fully automatic generation of mappings from different ontology information is generally considered impractical [klein, noy]. This is because there is a degree of uncertainty in any automatic approach to matching two ontologies, with this uncertainty caused by the different syntactic representation of the ontologies, the combination of the similarity measures produced by different matchers, and the heuristic approaches inherent in some matchers [cross]. For now, semi-automatic techniques for creating mappings from ontology matching information will continue to dominate [uschold].

As an example, attempts were made to semantically match the semantics of the SMI and CIM network management models. It could be argued that the repeated use of mapping tools to undertake dynamic mapping between SMI and CIM models is not really necessary, as one definitive mapping should be possible for such a pair of standard models. However in reality due to vendor implementation, there is a great variety in models for these two standard areas and therefore a mapping tool should be beneficial. Therefore for this project, we wanted to investigate whether general purpose ontology mapping tools would be helpful in this task. For this project, the OISIN mapping framework [osullivan] was used to generate the mappings between the CIM and MIB ontologies. According to the Shvaiko and Euzenat classification mentioned above [shvaiko] Oisin incorporates a languagebased matcher, a linguistic resource matcher, a type-based matcher, and a reuse-based matcher. However, for this project the OISIN tools were used in a more traditional mode, where an expert assisted in the generation of the mappings at design time.

For this example, SMI information models, the ENTITY-MIB and HOST-RESOURCES-MIB were chosen to be semantically mapped to parts of the CIM object model. These models have the advantages to contain many common concepts expressed in different ways. Furthermore, the CIM model contains a number of mappingStrings attributes containing semantic information about some parts of the CIM model in terms of roughly equivalent SMI concepts.

In order for ontological mapping to be performed on the models, they each were first translated in an ontological format. It was necessary that this mapping was performed in manner where the loss of semantics incurred by such a mapping was minimized. Research from the Universidad Politécnica de Madrid (UPM) [lópezdevergara02] describes how such an ontological translation can be performed, where a plugin was created for the Protégé ontology editor, allowing SMI MIB files and CIM MOF files to be imported into the editor in a .standardized model. In the work described in this paper, this plugin tool was used for the conversion of the CIM object-oriented model of classes and properties to an ontological model. However, the plugin for the SMI MIB files did not function in a manner that produced an ontology usable for this research. Consequently an XSLT transformation of an XML representation of the MIB files produced a suitable ontology. The XML representation of the MIB was produced using the libsmi library, available from the Technical
University

of

Braunschweig

(http://www.ibr.cs.tu-bs.de/projects/libsmi). Once these ontologies were created, a number of methods for automated and semi-automated semantic matching were attempted, as shown in Figure 2.

Initially the lexical matcher in OISIN was used over the two ontologies. However this resulted in a large number of matches that made it difficult to identify mappings due to the volume of candidates. Upon investigation it was found that the large volume of candidate matches was due to the frequent use of the same substrings in property terms. For example, in the HOST-RESOURCES-MIB there is a class called hrPrinterEntry with a property hrPrinterStatus. The UPM tool appends the prefix "CIM_Printer" to classes and properties as

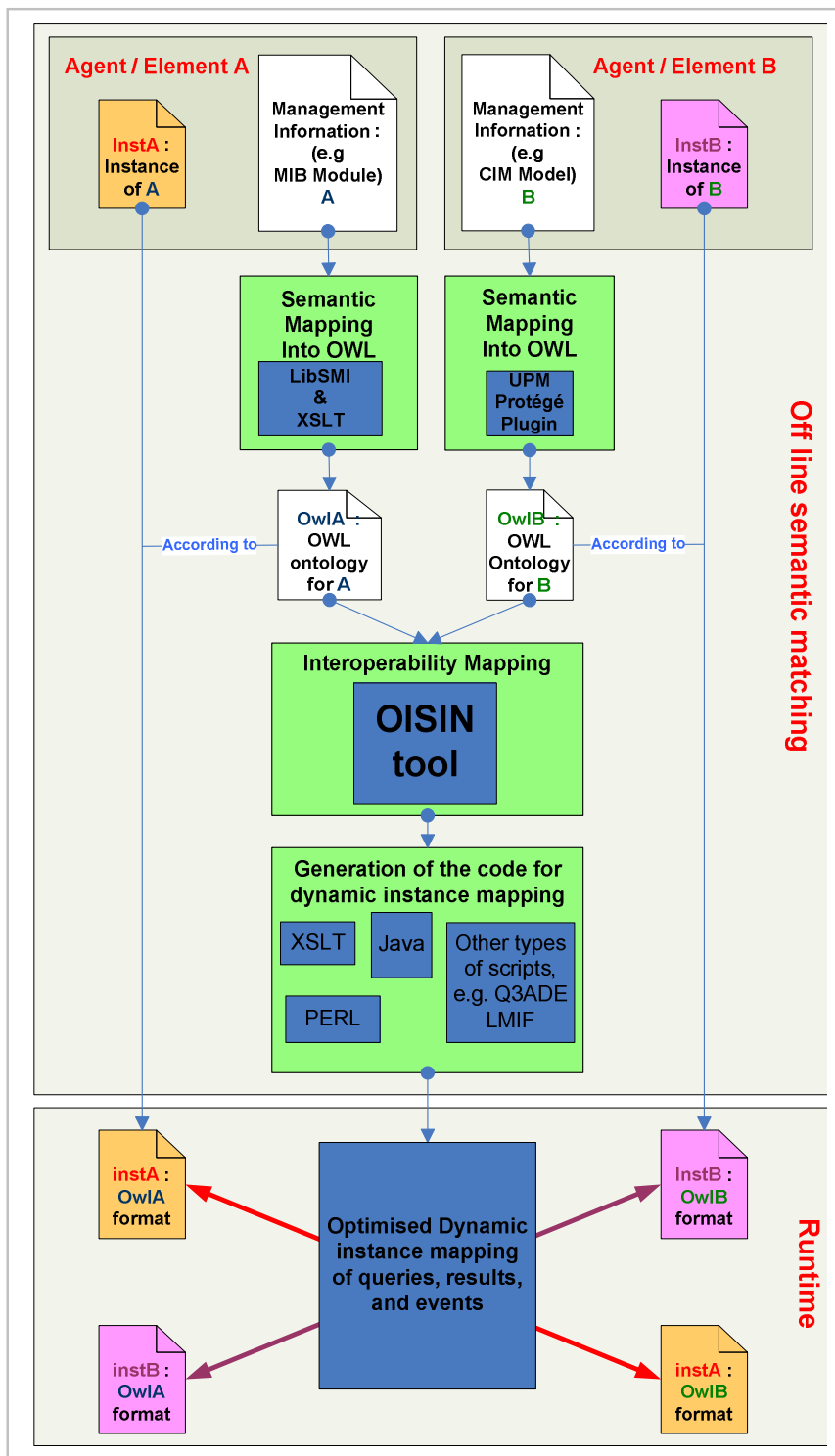

Figure 2: Combination of offline and runtime semantic matching 
the ontology is being created from the CIM definition. This results in the hrPrinterStatus property lexically matching to thirty seven properties in the CIM ontology, thirty matching to the "Printer" prefix and seven matching to "Status" part of the term. Even removing the "CIM_Printer" prefix that had been added by the UPM tool results in ten matches for that one property. Although this is more manageable it is still places unacceptably large volume of potential mapping candidates for a user. In addition, telecom specific acronyms or usage of terms were unknown by the general purpose online dictionary used and this resulted in some matches being missed. For example, the term "cluster". A combination of these issues meant that the matches presented in the user interface were not helpful in the identification of mappings. To overcome the problem of using a general purpose lexical matcher, it was decided to develop a CIM/MIB specific matcher that could be used in conjunction with the lexical matcher to improve the identification of candidate matches. The introduction of this domain specific matcher was straightforward as the OISIN framework is open and extensible. Initially the matcher has been designed to take advantage of the mappingStrings qualifier of the CIM MOF files. The matcher queries XML versions of the MOF files associated with the CIM ontology in question to build up a list of CIM property terms and mappingStrings qualifier value where this exists. For example, the property Availability of CIM_LogicalDevice has "MIB.IETF|HOST-RESOURCES-MIB.hrDeviceStatus" as the mappingStrings qualifier value. The ontologies are then examined to see if these terms appear. Where they do, a match is created and a strength value for the match assigned. The strength of the match is a numeric weighting that is assigned by the matcher. The results of the domain matcher and lexical matcher are then processed by a matcher combination component within OISIN to calculate an overall strength of matches for the ontologies. The overall match result is presented to the user in the graphical user interface, with different strength matches being highlighted using different colors. Of course, the outcome of the application of the CIM/MIB matcher can be quite variable as it depends on the occurrence of mappingStrings qualifier in the CIM model being analysed. For example for the HOST-RESOURCES CIM ontology discussed in this paper, only eighteen out of one hundred and seventy six mappingString qualifiers were specified.

From this initial investigation, it is clear that lexically based matching approaches alone will not be sufficient for assisting the identification of mappings between management ontologies, and the use of additional domain information such as the mappingString qualifier is of limited benefit. Therefore what kind of functionality needs to be added to general purpose ontology mapping frameworks for this domain requires further investigation and experimentation. The answer may lie in a reuse orientated approach where an expert creates the mappings for a pair of models once based on the standards. The ontology mapping functionality that is required relates to sharing these reusable mappings and the identification of where the ontologies deviate from the standard model such that the focus for mapping can be placed there.

\section{B. The Knowledge Delivery Service and Knowledge Delivery Network}

The Knowledge Delivery Service acts as an interface to the Knowledge Delivery Network. As stated the KDN is assembled as a Content Based Network to exploit both the publish-subscribe nature of the network and its ability to perform advanced filtering on the contents of each message. Semantic interoperability mappings are performed in both the KDS and the KDN.

Firstly, subscriptions to the KDN will be represented in a number of different models by different subscribers. To enable matching to occur between these different types of consumer subscriptions and the different advertisements provided by knowledge producers, the subscriptions and notifications must be presented according to a model understood by the subscription matcher. To facilitate this matching the subscription matching mechanism will need to be extended to support more complex ontological functions such as equivalence and subsumption. In addition the KDN will be extended to address consumption of composite notifications [courtenage].

The KDS will meanwhile be responsible for the semantic mappings, between the different models used for events, knowledge queries, and their responses. Using these mappings instances of events, queries, and responses must be dynamically translated to allow a knowledge consumer to receive messages in a format it can understand, rather than the format used by knowledge producer (if different). As stated this mapping is driven by optimized runtime mappings generated from the offline semantic interoperability matching between information models as described above. In this way the considerable overhead resulting from ontology reasoning tools is avoiding during runtime, while still exploiting the power of ontology-based semantics.

\section{RUNTIME INTEROPERABILITY AND FINDINGS}

In this section we present some performance evaluations to give an indication of the suitability of our proposed solution for a knowledge delivery service to the telecommunication management domain.

To implement a proof of concept scenario we have assembled an initial prototype as an experimental testbed. It consists primarily of:

- Elvin, a commercially available CBN solution [segal]

- Q3ADE, a commercial network element management platform with extensive support for constructing gateways between different management protocols [uhc].

From the design described in Figure 3 a proof of concept architecture was assembled. A knowledge consumer declares an interest in network events from which a CBN subscription is created. At the other end of the network, an SNMP agent 
monitors some resource, initiating SNMP Trap messages as required. These Traps are detected by a Q3ADE element management agent, and internally translated to ITU X.733 internet alarms [itu]. A scripted event forwarding discriminator (EFD) within the Q3ADE platform allows these events to be filtered and forwarded to a message consumer. These alarms are further filtered and processed, for example extracting information such as the probable cause of the event and the perceived severity of the alarm. A complete

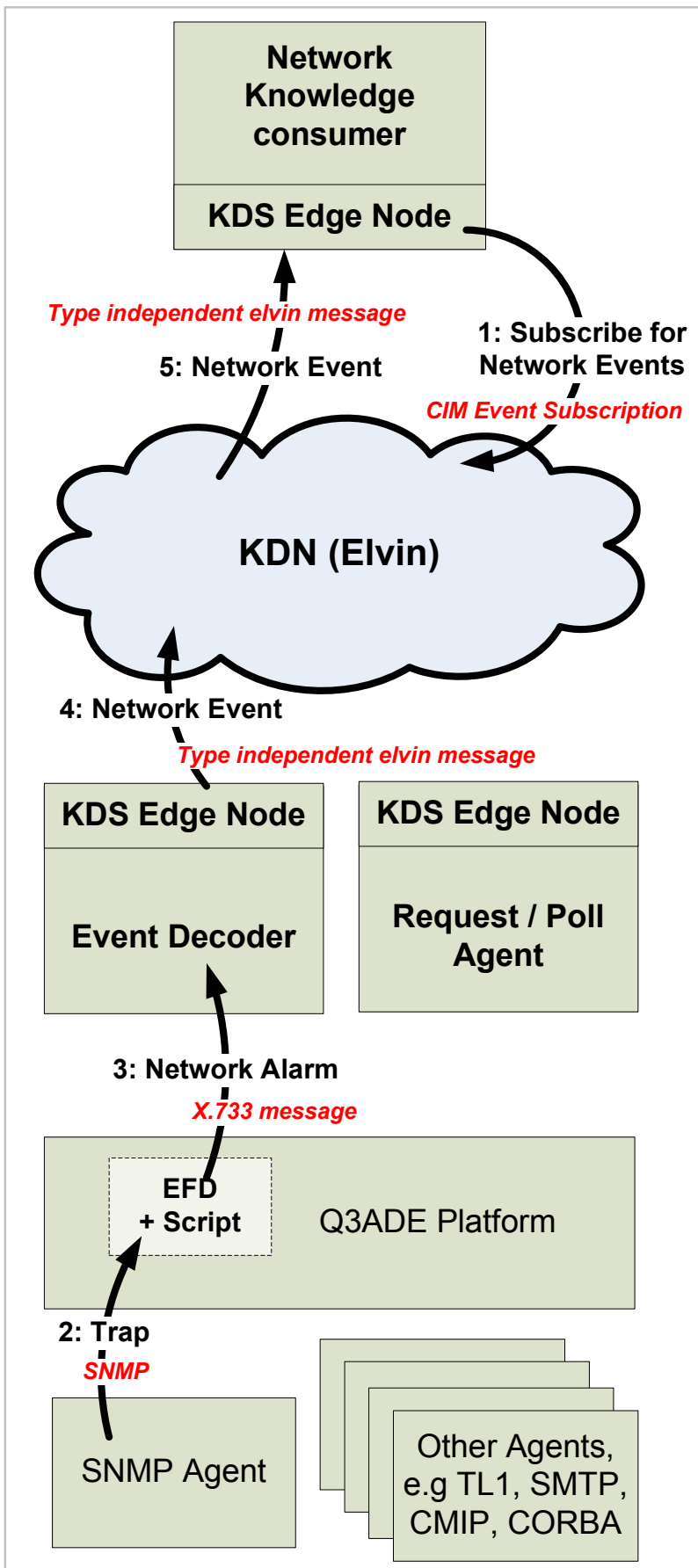

Figure 3: Prototype Knowledge Delivery Service description of the alarm is then incorporated into a notification and passed to the KDN. As described, the KDN is currently implemented as an Elvin Content Based Network. Within the KDN a matching algorithm dynamically routes the message to the consumer in a format that can be understood without support for decoding SNMP Trap PDUs. The performance of this part of the case study implementation is described in later in this section.

In addition to support for asynchronous messaging, the consumer may wish to either query or poll a network element or group of network elements. The framework described in Figure 3 supports dynamic subscription to network events and the dynamic creation of model independent queries and polls. The mapping between the different information models is achieved by table lookup routines based on a set of mappings created as described in section IIIA of this paper.

As a concrete example, a consumer may express interest in receiving information about devices, in the form of a CIM query (e.g. CIM_Printer). By exploiting the ontological mappings described in the previous section, this query is automatically satisfied by a SNMP query for devices described as hrPrinterEntry [lewis05], described in the SMI HOST-RESOURCES-MIB. This process is shown in Figure 3 whereby the mappings extracted from the ontological semantic matching are queried, the request is assembled and passed to the KDN. Elsewhere in the KDN other nodes receive the query and pass back the information requested in a format dependent on its own management information model. Within the KDS the result is translated back to the format required by the consumer. This mechanism allows the consumer to monitor the network, and extract knowledge from it, without the necessity to agree on a representation model for that knowledge, and without the need to address individual elements or agents.

We have used the Q3ADE platform to develop a gateway between SNMP and the Elvin CBN. This gateway currently accepts SNMPv2 traps from an SNMP agent and converts them to Elvin notification messages. Thus the gateway acts as a CBN event producing client. Q3ADE provided a powerful platform for rapidly developing this gateway as it already implemented proprietary text-based protocol whose encoding could readily be used in constructing Elvin messages.

\begin{tabular}{|c|c|}
\hline $\begin{array}{c}\text { Average end to end time } \\
\text { for Standard TRAP } \\
\text { messages }\end{array}$ & $\begin{array}{c}\text { Average end to end time } \\
\text { messages transmitted using } \\
\text { the KDS }\end{array}$ \\
\hline $0.607 \mathrm{~ms}$ & $29.06 \mathrm{~ms}$ \\
\hline
\end{tabular}

Table 1: Comparing KDS latency to SNMP latency

A sample industrial grade network element management system may create events at a rate of between 2 events per second in normal operation, and up to 15 events per second when in an overload condition. At all times event latency (end

1 All measurements were carried out on a single machine to facilitate the measurement of processing load rather than network load. (Intel ${ }^{\circledR}$ Pentium ${ }^{\circledR} \mathrm{M} 2 \mathrm{GHz}, 1 \mathrm{~GB}$ RAM) 


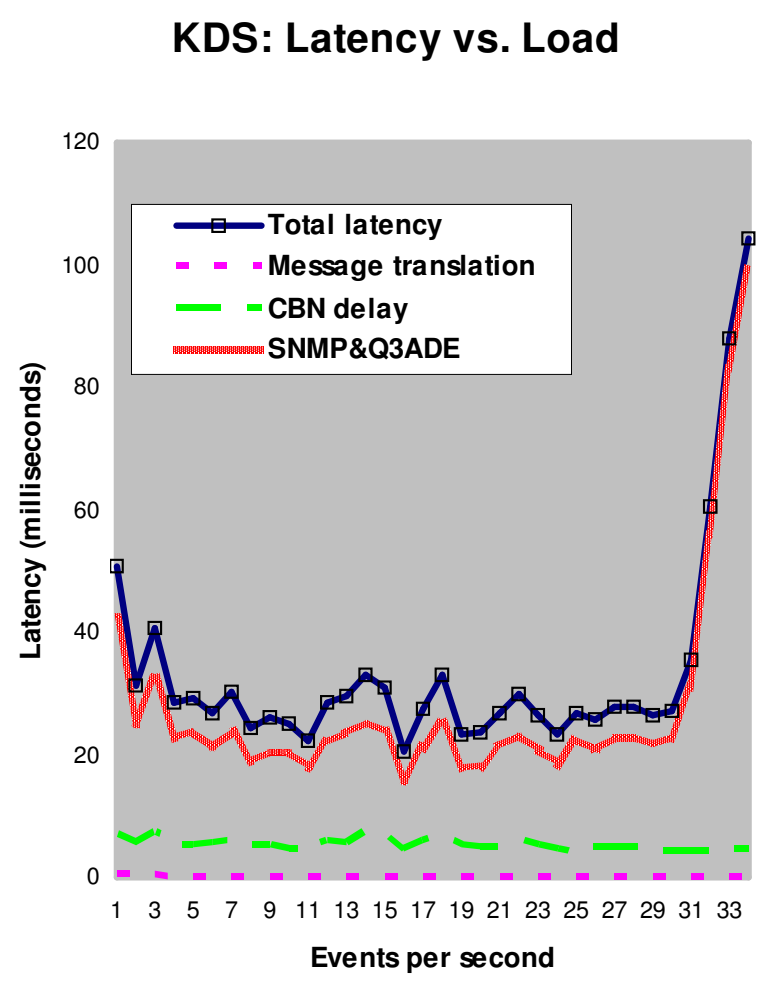

Figure 4: KDS: Latency vs. Load

to end) must be less than 2 seconds. Although the results presented in Table 1 above represent only processing time on a single machine ${ }^{1}$, because the packet size of each event is very small by comparison to the capacity of even medium speed networks, the results above fall well within these specific industrial grade requirements. In fact the degradation of performance of the KDS at approximately 30 messages per second, as seen in Figures 4 and 5 , is not due to communication overhead, instead it is mostly due to the memory and processing bottlenecks that arise from all of the software operating on one machine, therefore demonstrating significant potential for improvement if the KDS was distributed as intended.

\section{CONCLUSIONS AND FURTHER WORK}

This paper described a framework for the delivery of network knowledge as context for decision making in various forms of management solutions, from centralized human-driven ones, to decentralized ones based on autonomous intelligent agents. At both extremes there is a need to deal with the semantic mismatches that may arise between the knowledge understood by the (human or automated) entity making management decisions and the wide variety of knowledge on the state of the network available from the network elements. This framework addresses this problem by using ontological forms of the

\section{KDS Latency vs. SNMP latency}

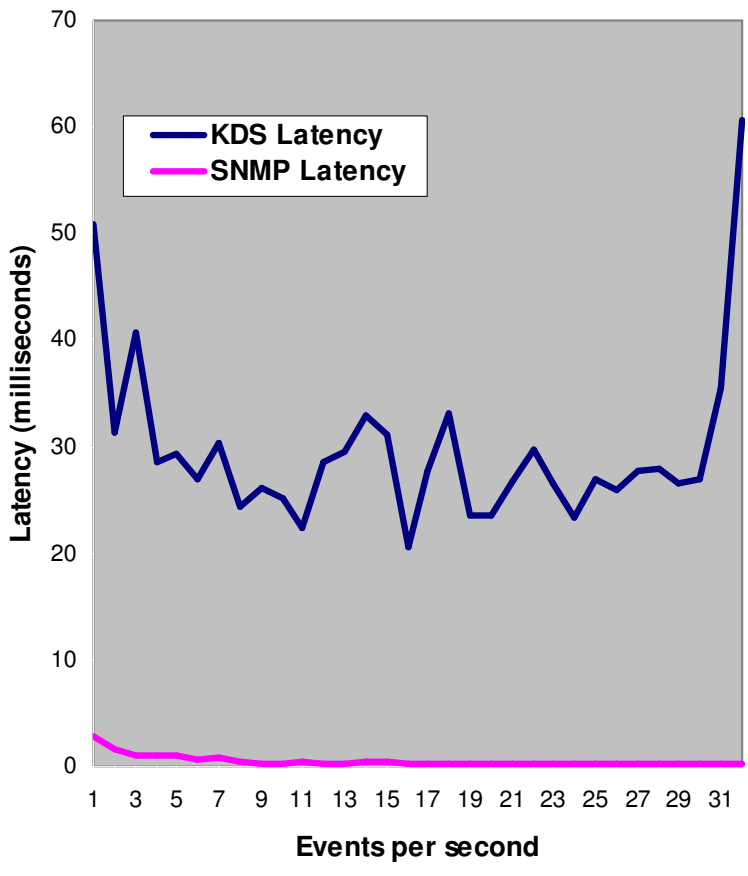

Figure 5: KDS Latency vs. SNMP Latency

management information models that represent this knowledge and using automated aids, such as lexical and structural mapping to assist human interoperation engineers identify possible mappings between elements of different models. As a case study of our approach we focus on the delivery of notifications from network elements to decision making entities, for which a content based network is used. This delivers notifications as messages of type-value pairs expressed as strings that are matched to subscriptions from managers. This allows us to readily deploy semantic mapping transforms in a number of ways, generating these transforms from any semantic interoperability mappings identified. In this way the benefits of ontologies in semantic interoperability can be deployed in management systems without having to introduce demanding ontological reasoning into the runtime delivery path.

Many issues still need to be addressed to make this approach a practical reality. Principle among these is the already introduced issue of access control. In any multi-domain scenario those responsible for any knowledge resource must be able to impose control over who is able to access that knowledge. Access control policies have been demonstrated to work with CBN sources [belokosztolszki]. This employed role-based access control which is the predominant approach to defining access control policies. However, in a fluid, multidomain scenarios the detailed business modeling that underpins the identification of the roles used will make this 
approach very brittle. Instead this project will adopt a community-based policy approach, which has been shown to track more easily and accurately the dynamic organizational grouping within and between organizations [feeney04]. This approach will be adapted to support access control of knowledge available to the KDN, including a mechanism to identify possible access control conflicts and suggest resolutions. Such KDN access control requires new mechanisms for matching subscriptions to producer access control rules, without strongly binding subscriptions to consumer attributes and thereby reducing the routing efficiency gained by subscription aggregation. This will be combined with a trust-based access control mechanism for determining community membership in ad hoc organizational situations [feeney05].

Another issue that we have not yet fully assessed in the management domain is the level of useful semantic interoperability mapping can be discovered for the existing body of management information models, both open and proprietary. Improved information model to ontology conversion tools, as well more advanced ontology mapping tools are required, along with a concerted effort from industry bodies in creating inter-model mappings and supporting conversion.

\section{ACKNOWLEDGMENT}

This work was supported partially by Science Foundation Ireland and Enterprise Ireland through the Centre for Telecommunication Value Chain Research. The authors would like to thank Jorge E. López de Vergara and Víctor A. Villagrá of UPM for allowing access to their tools for converting management information models. The authors would also like to thank UH Communications $\mathrm{A} / \mathrm{S}$ for allowing access to their Q3ADE platform.

\section{REFERENCES}

[adams] Adams, E., Willetts, K., "The Lean Communication Provider: Surviving the Shakeout through Service Management Excellence", McGrw-Hill, 1996

[belokosztolszki] Belokosztolszki, A., Eyers, D.M., Pietzuch, P.R., Bacon, J., Moody, K., "Role-based access control for publish/subscribe middleware architectures". In International Workshop on Distributed Event-Based Systems (DEBS03), ACM SIGMOD, San Diego, CA, USA, 2003. ACM.

[carzaniga ] Carzaniga, A., Rosenblum, D. S., and Wold, A. L., The Design and Evaluation of a Wide-Area Event Notification Service, ACM Transactions on Computer Systems, Vol. 19, Issue 3, August 2001

[chand] Chand, R., Felber, P.A., "A Scalable Protocol for ContentBased Routing in Overlay Networks", Second IEEE International Symposium on Network Computing and Applications, April 2003, Cambridge, MA

[cim] Common Information Model v2.5, DMTF 2000: http://www.dmtf.org/spec/cim_schema_v25.html [clark] D. Clark, C. Partridge, J.C. Ramming, J. Wroclawski, "A Knowledge Plane for the Internet", SIGCOMM'03

[courtenage] Courtenage, S. "Specifying and Detecting Composite Events", In 1st International Workshop on Discrete Event-Based Systems, Vienna, 2002

[cross] Cross, V., "Uncertainty in the automation of ontology matching". Fourth International Symposium on Uncertainty Modeling and Analysis, 2003. ISUMA 2003. 135- 140, September 2003.

[crowcroft] Crowcroft, J., Jean Bacon, J., Pietzuch, P., Coulouris, G., Naguib. H., "Channel Islands in a Reflective Ocean: Large-Scale Event Distribution in Heterogeneous Networks", IEEE Communications Magazine, 40(9):112-115, September 2002.

[duke04] Duke, A., Davies, J., Richardson, M., Kings, N., "A Semantic Service Oriented Architecture for the Telecommunications Industry", in proc of IFIP Int'l Conf on Intelligence in communications Systems, Bangkok, Thailand, Nov 2004 (INTELLICOM 2004), Springer LNCS 3283, pp 236-245

[feeney04] Feeney, K., Lewis, D., Wade, V., "Policy based Management for Internet Communities", Proceeding of the 5th IEEE International Workshop on Policies for Distributed Systems and Networks, 7-9 June 2004, York Town Hieght, NY, USA

[feeney05] Feeney, K., Quinn, K., Lewis, D., O'Sullivan, D., Wade, V. "Relationship-Driven Policy Engineering for Autonomic Organizations", to appear in proa of 6th IEEE International Workshop on Policies for Distributed Systems and Networks, 2005

[loser] Loser, A., Naumann, F, Siberski, W., Nejdl, W., Thaden, U, "Semantic Overlay Clusters within Super-Peer Networks". Proceedings of the International Workshop on Databases, Information Systems and Peer-to-Peer Computing in Conjunction with the VLDB 2003.

[lópezdevergara04] López de Vergara, J.E., Villagrá, V.A., Berrocal, J., "Applying the Web Ontology Language to management information definitions", IEEE Communications Magazine, Vol. 42, Issue 7, July 2004, pp. 68-74. ISSN 0163-6804

[lópezdevergara02] López de Vergara, J.E., Villagrá, V.A., Berrocal, $\mathrm{J}$, "Semantic Management: advantages of using an ontology-based management information meta-model", Proceedings of the HP Openview University Association Ninth Plenary Workshop (HPOVUA'2002), distributed videoconference, 11-13 June 2002

[martin] David Martin, Mark Burstein, Jerry Hobbs, Ora Lassila, Drew McDermott, Sheila McIlraith, Srini Narayanan, Massimo Paolucci, Bijan Parsia, Terry Payne, Evren Sirin, Naveen Srinivasan, Katia Sycara, "OWL-S: Semantic Markup for Web Services", W3C Member Submission 22 November 2004

[itu] ITU Recommendation X.733 / ISO/IEC 10164- 4, "Information Technology - Open Systems Interconnection - System Management: Alarm Reporting Function”, 1992

[kalfoglou] Y., Kalfoglou, Schorlemmer, W.M., "Ontology Mapping: The State of the Art.", in Dagstuhl Seminar Proceedings: Semantic Interoperability and Integration 2005, http://drops.dagstuhl.de/opus/volltexte/2005/40

[kephart] J. Kephart, D. Chess "The Vision of Autonomic Computing" Computer, Jan 2003

[klein] M. Klein, "Combining and relating ontologies: an analysis of problems and solutions". In Workshop on Ontologies and Information Sharing, IJCAI'01, Seattle, USA, August 4-5, 2001

[lewis05] Lewis, D., O’Sullivan, D., Power, R., Keeney, J., "Semantic Interoperability for an Autonomic Knowledge Delivery Service". To be presented at the 2nd IFIP TC6 International Workshop on Autonomic Communication (WAC 2005). Oct. 3-5, 2005, Vouliagmeni, Athens, Greece 
[mullany] Mullany, F., Ho, L., Samuel, L., Claussen, H., "SelfDeployment, Self Configuration: Critical Future Paradigms for Wireless Access Networks", in Proc of the 1st IFIP International Workshop on Autonomic Communications, Dec 2004

[noy] Noy, N.F., Musen, M.A., "Anchor-PROMPT: Using NonLocal Context for Semantic Matching”. Workshop on Ontologies and Information Sharing at the Seventeenth International Joint Conference on Artificial Intelligence (IJCAI-2001), Seattle, WA, 2001.

[osullivan] D. O'Sullivan, D. Lewis, Semantically Driven Service Interoperability for Pervasive Computing, Proc. 3rd ACM International Workshop on Data Engineering for Wireless and Mobile Access, San Diego, USA, 19 Sept 2003, ACM Press.

[owl] OWL Web Ontology Language Reference, available at: http://www.w3.org/TR/owl-ref/.

[schulzrinne] Schulzrinne, H., Hancock, R., "GIMPS: General Internet Messaging Protocol for Signaling", Internet Draft, draftietf-nsis-ntlp-05, February 2005

[segall] Segall, B. et al, "Content-Based Routing in Elvin4", In Proceedings AUUG2K, Canberra 2000.

[shvaiko] Shvaiko, P. Euzenat, J., "A survey of schema-based matching approaches. Technical Report DIT-04-087, University of Trento, October 2004 [smirnov] Smirnov, M.: "Autonomic Communication: Research Agenda for a New Communication Paradigm", in Fraunhofer FOKUS White Paper, 2004.

[strom] Strom et al., "Gryphon: An Information Flow Based Approach to Message Brokering“" In International Symposium on Software Reliability Engineering 1998 [smirnov] Smirnov, M., "Autonomic Communications: A Research Agenda for a New Communication Paradigm", November 2004, http://www.autonomiccommunication.org/publications/doc/WP_v02.pdf

[thaler] Thaler, D. G., Ravishankar, C.V., "An Architecture for Interdomain Trouble Shooting", Journal of Network and Systems Management, 12(2), pp 155-189, June 2004

[tmf053] NGOSS Architecture, Technology Neutral Specification, Membership Evaluation Version 1.51, TeleManagement Forum, July 2001

[tmn] ITU-T Recommendation M.3010 (1992), Principles for a TMN [uhc] UHCommunications A/S, The Q3ADE Platform. http://www.uhcommunications.com/products.htm

[uschold] Uschold, M., Grüninger, M., "Ontologies and Semantics for Seamless Connectivity". SIGMOD Record 33(4): 58-64. December 2004.

[wsmo]. Web Service Modeling Ontology (WSMO) D2v1.1, February 2005, http://www.wsmo.org/2004/d2 